\title{
Clinical characteristics of patients hospitalized for ocular chemical injuries in Shanghai from 2012 to 2017
}

\author{
Tao Li $\cdot$ Bo Jiang $\cdot$ Xiaodong Zhou
}

Received: 8 June 2019/ Accepted: 28 December 2019/Published online: 9 January 2020

(C) The Author(s) 2020

\begin{abstract}
Purpose To summarize the clinical characteristics of patients with ocular chemical injuries and evaluate their potential relationship with the visual outcome by analyzing the medical records of these patients from January 1, 2012, to December 31, 2017.

Methods This is a retrospective case series study. Patient data included age, gender, occupational classification, location of ocular chemical injury, initial and final best-corrected distance visual acuity (BCDVA), intraocular pressure (IOP), nature and chemical phase, distribution and severity of chemical injury, management methods, and complications. All variables were evaluated for their potential relationship with visual outcome.
\end{abstract}

Tao Li and Bo Jiang contributed equally to this work.

T. Li $\cdot$ B. Jiang $\cdot$ X. Zhou $(\bowtie)$

Department of Ophthalmology, Jinshan Hospital of Fudan University, 1508 Longhang Road, Shanghai 201508,

China

e-mail: xdzhou_2013@163.com

T. Li

e-mail: litao13013@sina.com

B. Jiang

e-mail: jb19811119@sina.com

T. Li

Department of Ophthalmology, Eye \& ENT Hospital of Fudan University, Shanghai, China
Results A total of 160 patients were hospitalized with ocular chemical injuries. Majority of the patients were factory workers and arrived at the consultation room less than $24 \mathrm{~h}$ after injury. The most common ocular injury setting, classification of severity, causative chemical, chemical phase, and complications were workplace, grade II, unknown and mixed substance, liquid, and elevated IOP, respectively. The risk factors for poor final BCDVA were identified as older age, poor initial BCDVA, and irrigation $24 \mathrm{~h}$ after injury $(P<0.001, P<0.001$, and $P=0.011$, respectively).

Conclusions We elaborate the clinical characteristics and outcomes of patients with ocular chemical injuries in Jinshan District, Shanghai. A comprehensive education program should be established and the use of protective eyewear should be promoted to prevent occupation-related ocular chemical injuries.

Keywords Clinical characteristic Ocular chemical injury · Ocular surface

\section{Introduction}

Ocular chemical injuries account for more than $10 \%$ of all ocular traumas $[1,2]$. These injuries may lead to long-term visual impairment, the likelihood of which has been associated with the initial extent of injury. Most victims are men aged 20-40 years, and the injury 
commonly occurs in industrial chemical factories or at home [3, 4]. Furthermore, these conditions can result in a significant economic burden to families and countries due to the time lost at work, the need for family care-giving, expensive hospital costs, prolonged follow-up, visual rehabilitation, and ocular plastic surgery [4]. Therefore, patients at high risk of chemical injuries should be reminded to take every precaution and ensure prompt treatment. To date, only a few studies have explored the clinical features in patients with ocular chemical injuries [5-7]. An assessment of clinical characteristics of patients with ocular chemical injuries in Shanghai is essential.

Jinshan Hospital of Fudan University, formerly known as Shanghai General Petrochemical Factory Hospital, was established in 1975. The institute is located at Jinshan District in Shanghai City, one of the important chemical industrial areas in East China. It serves a population of more than 800,000 , and its specialty is the treatment of chemical injury. Although some ocular chemical injuries may be treated in general casualty clinics or other hospitals, the majority of ophthalmic patients usually come to this hospital.

The present study reviewed all hospitalized patients with an ocular chemical injury who were admitted to the Department of Ophthalmology, Jinshan Hospital of Fudan University, from January 1, 2012, to December 31, 2017. The aim of this study was to summarize the clinical characteristics of patients with ocular chemical injuries and evaluate their potential relationship with the visual outcome by retrospectively analyzing the medical records of these patients.

\section{Methods}

This study retrospectively collected data from all patients with a principal diagnosis of ocular chemical injury (ICD code: T26) who were hospitalized at the Department of Ophthalmology, Jinshan Hospital of Fudan University, China, from January 1, 2012, to December 31, 2017. All patients were diagnosed according to the International Classification of Diseases, Tenth Revision, Clinical Modification (ICD-10$\mathrm{CM}$ ). This study was approved by the Ethics Committee of Jinshan Hospital of Fudan University. All procedures conformed to the tenets of the Declaration of Helsinki. Written informed consent was obtained.
All patient data from the medical record system was examined including age, gender, occupational classification, location of ocular chemical injury, initial and final best-corrected distance visual acuity (BCDVA), nature and chemical phase, distribution and severity of injury, management methods, and complications. BCDVA was recorded with Snellen chart. Final BCDVA was obtained at the 1-month follow-up. The intraocular pressure (IOP) was determined using noncontact tonometry (CT-80; Canon Inc., Tokyo, Japan). The elevated IOP was defined as IOP $>21$ $\mathrm{mmHg}$. The severity of ocular chemical injury was graded by the extent of corneal haze and limbal ischemia according to the Roper-Hall classification [8]. Briefly, grade I was defined as corneal epithelial damage without limbal ischemia; grade II as corneal haze and visible iris details with less than one-third limbal ischemia; grade III as total corneal epithelial loss, stromal haze, and obscured iris details with more than one-third but less than half limbal ischemia; and grade IV as opaque cornea and obscured iris and pupil with more than half limbal ischemia. The management methods included immediate irrigation after injury, amniotic membrane transplantation (AMT), and topical medication (e.g., corticosteroid and prophylactic antibiotics).

Statistical analysis was performed using SPSS version 17.0 software (SPSS Inc., IL, USA). Demographics and patient characteristics were reported as the means and standard deviations or percentages in each group and included age, BCDVA, laterality of eyes, occupation, and location where the injury occurred. The independent $t$ test was used to evaluate differences in BCDVA and IOP before and after treatment. A one-way analysis of variance was used to evaluate differences in parametric variables among various causative chemical groups. All variables, including age, gender, delay in presentation, initial BCDVA, nature and condition of injury, irrigation, grade, AMT, and complications, were entered into the multivariate logistic regression analysis. All $P$ values were two-sided and considered statistically significant when less than 0.05 . 


\section{Results}

Patient demographics

A total of 160 patients were hospitalized with ocular chemical injuries from January 1, 2012, to December 31,2017 , when 5425 patients with the ocular disease were admitted to the Ophthalmology Department, Jinshan Hospital of Fudan University. Therefore, ocular chemical injury accounted for approximately $2.9 \%$ of hospital admissions each year.

The demographics of these subjects are shown in Table 1. Among these patients, $136(85 \%)$ were males, with a male-to-female ratio of 5.7:1. The mean age of the patients was $42.8 \pm 12.7$ years, ranging from 19 to 74 years. No significant difference in age was found between genders $(P=0.546)$, with a mean age of $42.5 \pm 12.1$ years in males and $44.2 \pm 16.0$ years in females. The age distribution showed that the peak occurrence of ocular chemical injury occurred in the 41- to 50-year group ( $n=55$; $34.4 \%)$. The highest proportion in males $(n=50$; $36.8 \%$ ) was also distributed in the 41 - to 50 -year

Table 1 Prevalence of ocular chemical injuries in Jinshan District, Shanghai City $(n=160)$

\begin{tabular}{ll}
\hline Agent & Patients \\
\hline Age (years) & $42.8 \pm 12.7$ \\
Genders (\%) & $136(85.0)$ \\
Male & $24(15.0)$ \\
Female & \\
Laterality of eyes (\%) & $42(26.3)$ \\
OD & $41(25.6)$ \\
OS & $77(48.1)$ \\
OU & \\
Occupation (\%) & $126(78.8)$ \\
Factory & $10(6.3)$ \\
Construction & $24(15.0)$ \\
Other & \\
Location where injury occurred $(\%)$ & $137(85.6)$ \\
Workplace & $14(8.8)$ \\
Home & $6(3.8)$ \\
Beauty parlor & $2(1.3)$ \\
Restaurant & $1(0.6)$ \\
Swimming pool &
\end{tabular}

group, while that in females was in the 31- to 40-year group ( $n=6,25.0 \%)$ (Fig. 1).

A total of $41(25.6 \%)$ patients with right eye involvement, $42(26.3 \%)$ patients with left eye involvement, and $77(48.1 \%)$ patients with bilateral eye involvement were included. The initial BCDVA in the affected eye was $0.38 \pm 0.25$, and the IOP was $17.6 \pm 4.0 \mathrm{~mm} \mathrm{Hg}$. The majority of the patients were factory workers $(n=126 ; 78.8 \%)$, followed by construction workers $(n=10 ; 6.3 \%)$. The most common ocular injury setting was workplace in 137 patients (85.6\%), followed by home in 14 patients (8.8\%). None of the patients wore protective goggles at the time of injury.

As shown in Fig. 2, the majority of patients $(n=133,83.1 \%)$ presented for treatment within $24 \mathrm{~h}$ of injury: $64(40.0 \%)$ less than $3 \mathrm{~h}, 18(11.3 \%)$ within 3-6 h, $10(6.3 \%)$ within 6-12 h, and 41 (25.6\%) within 12-24 h. The longest delay in presentation was $552 \mathrm{~h}$.

The most common classification of the severity of ocular chemical injury was grade II in 116 eyes $(48.9 \%)$ of 79 patients $(49.4 \%)$, followed by grade I in 64 eyes (27.0\%) of 41 patients (25.6\%) (Fig. 3).

Nature and chemical phase of ocular injury

A large number of chemicals were involved in these injuries. The most common causative chemical was unknown and mixed substances in 90 eyes (38.0\%) of 58 patients $(36.3 \%)$, followed by plural acid in 59 eyes

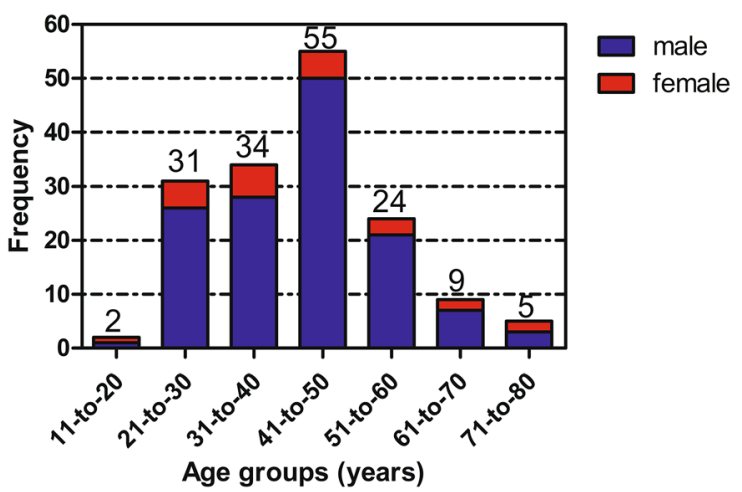

Fig. 1 Frequency of ocular chemical injury by age and gender. The age distribution showed that the peak occurrence of ocular chemical injury occurred in the 41 - to 50-year group $(n=55$; $34.4 \%)$. The highest proportion in males $(n=50 ; 36.8 \%)$ was also distributed in the 41- to 50-year group, while that in females was in the 31 - to 40 -year group $(n=6 ; 25.0 \%)$ 


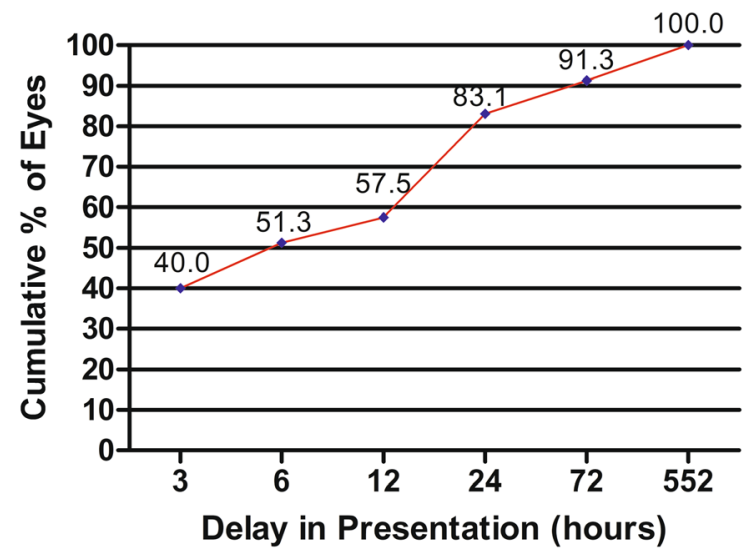

Fig. 2 Percentage of patients arriving at the consultation room for medical treatment

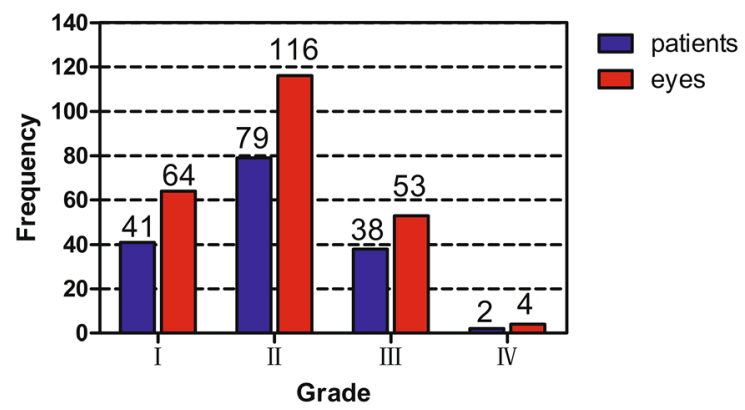

Fig. 3 Frequency of ocular chemical injury using the RoperHall classification. The most common classification of the severity of ocular chemical injury was grade II in 116 eyes (48.9\%) of 79 patients $(49.4 \%)$

(24.9\%) of 40 patients (25.0\%) (Fig. 4). As illustrated in Table 2, significant differences among the various causative chemical groups were found for initial BCDVA $(P=0.001)$. Post hoc analyses showed that initial BCDVA was significantly better in the organic chemical group than in the plural acid group and unknown and mixed substances group $(P=0.005$ and $P=0.035$, respectively). Initial BCDVA was also significantly better in the alkali agent group than in the plural acid group $(P=0.034)$. No other significant differences existed between groups (all $P>0.05$ ).

Moreover, the most common chemical phase was liquid in 191 eyes $(80.6 \%)$ of 132 patients $(82.5 \%)$, followed by a mixture of liquid and solid in 21 eyes (8.9\%) of 15 patients (9.4\%) (Fig. 4).
Treatment and prognosis of ocular chemical injury

Only 138 patients irrigated the affected eyes more than half an hour after injury, whereas the remaining 22 patients sought medical attention and dealt with the offending agents of the eyes until $24 \mathrm{~h}$ after injury. A total of 89 eyes underwent AMT surgery, including 2 surgeries for 3 eyes and 3 surgeries for 1 eye. The remaining 148 eyes were treated conservatively with medications. The final BCDVA in the affected eye was $0.70 \pm 3.0$. As also illustrated in Table 2, no significant differences among the various causative chemical groups were found for the final BCDVA $(P=0.651)$. The final BCDVA in the affected eyes was significantly better than the initial BCDVA in all causative chemical groups (all $P>0.001$ ). The complications are summarized in Table 3. The most frequent problem was elevated IOP in 20 eyes (8.4\%), followed by corneal opacity in 4 eyes $(1.7 \%)$.

Multivariate logistic regression analysis for potential factors associated with BCDVA

The risk factors for poor final BCDVA were identified as older age, poor initial BCDVA, and irrigation $24 \mathrm{~h}$ after injury (Table 4; $P<0.001, P<0.001$, and $P=0.011$, respectively). The final BCDVA was not significantly associated with the patient's gender $(P=0.615)$, delay in presentation $(P=0.525)$, nature of injury $(P=0.924)$, condition of injury $(P=0.332)$, grade $(P=0.198)$, or complications $(P=0.655)$. Its association with AMT $(P=0.069)$ was marginally significant.

\section{Discussion}

The present study described the characteristics and outcomes of 160 patients who were hospitalized with ocular chemical injuries at the Jinshan District in Shanghai over a 5-year period. A large number of patients presented to our hospital due to chemical injuries, where a large number of chemical enterprises were located nearby. The reported incidence ranges from 1.5 per 100,000 to 13.3 per 100,000 [ $4,6,9]$. In addition, 1480 cases suffering from chemical injuries were reported to the Victorian Poisons Information Centre in Australia, supporting a telephone-based advice service, but the incidence did not be calculated 
Fig. 4 Ocular chemical injuries grouped by nature and condition of causative chemical. The most common causative chemical was unknown and mixed substances in 90 eyes $(38.0 \%)$ of 58 patients (36.3\%). The most common condition of the injuries was liquid in 191 eyes $(80.6 \%)$ of 132 patients $(82.5 \%)$

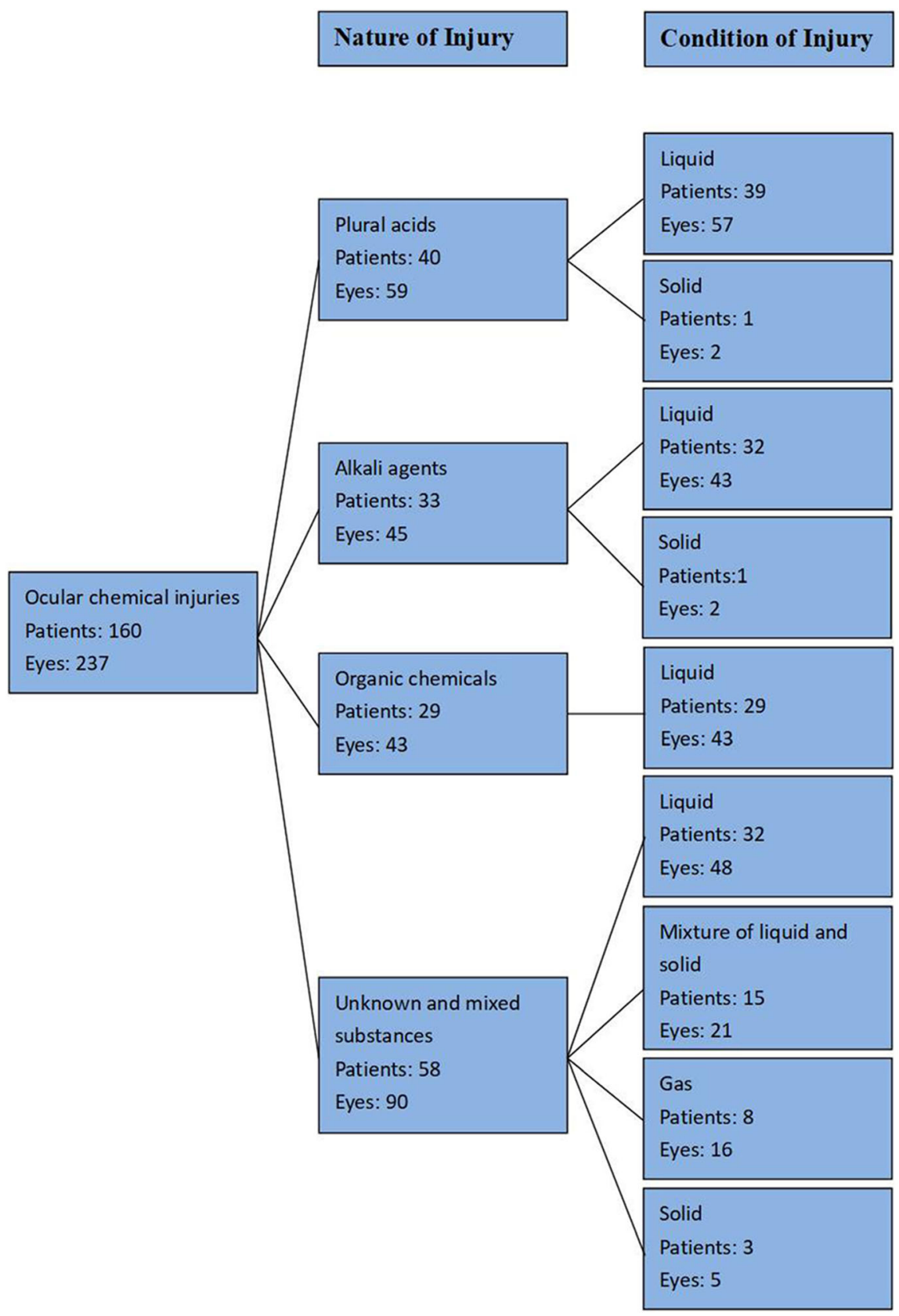

incidence of ocular chemical injuries might be underestimated due to the loss of patients who might have sought medical care in other hospitals and patients with minor injuries who received only outpatient treatment or declined admission.

In the present study, the mean age of the patients was $42.8 \pm 12.7$ years, which was in agreement with previous studies. Bizrah et al. [14] found that the mean 
Table 2 Clinical characteristics of ocular chemical injuries in various causative chemical groups in Shanghai $(n=239$ eyes)

\begin{tabular}{lllllll}
\hline & Treatment & Plural acids & Alkali agents & Organic chemicals & Unknown and mixed substances & $P$ value \\
\hline Age & - & $45.0 \pm 13.4$ & $40.5 \pm 10.8$ & $38.5 \pm 10.7$ & $44.6 \pm 13.7$ & 0.073 \\
Delay in presentation & - & $28.8 \pm 88.6$ & $14.2 \pm 15.1$ & $21.1 \pm 30.5$ & $35.3 \pm 52.2$ & 0.349 \\
BCDVA & Before & $0.31 \pm 0.23$ & $0.44 \pm 0.25$ & $0.48 \pm 0.29$ & $0.35 \pm 0.23$ & $0.001 * *$ \\
& After & $0.67 \pm 0.34$ & $0.72 \pm 0.26$ & $0.74 \pm 0.33$ & $0.70 \pm 0.29$ & 0.651 \\
IOP & Before & $17.9 \pm 3.9$ & $19.0 \pm 5.5$ & $16.3 \pm 3.2$ & $17.2 \pm 3.3$ & 0.102 \\
& After & $15.0 \pm 2.6$ & $15.5 \pm 3.1$ & $15.7 \pm 4.4$ & $16.0 \pm 3.1$ & 0.528 \\
\hline
\end{tabular}

$B C D V A$ best-corrected distance visual acuity, $I O P$ intraocular pressure

$* * P<0.01$

Table 3 Complication of ocular chemical injuries $(n=237$ eyes)

\begin{tabular}{lc}
\hline Complication $^{\dagger}$ & Eyes \\
\hline Elevated intraocular pressure & 20 \\
Corneal opacity & 4 \\
Symblepharon and entropion & 2 \\
Pseudopterygium & 1 \\
Conjunctival and scleral melt & 1 \\
Iridodialysis & 1 \\
Hypopyon & 1 \\
\hline
\end{tabular}

${ }^{\dagger}$ Some patients have more than two complications

Table 4 Multivariate logistic regression analysis for potential factors associated with final BCDVA

\begin{tabular}{lrc}
\hline Parameter & $B$ value & $P$ value \\
\hline Age & -0.007 & $<0.001^{* * *}$ \\
Gender & 0.029 & 0.605 \\
Delay in presentation & 0.000 & 0.525 \\
Initial BCDVA & 0.338 & $<0.001^{* * *}$ \\
Nature of injury & 0.002 & 0.924 \\
Chemical phase & 0.022 & 0.332 \\
Irrigation & -0.149 & $0.011^{*}$ \\
Grade & -0.039 & 0.198 \\
AMT & 0.075 & 0.069 \\
Complications & -0.037 & 0.655 \\
\hline
\end{tabular}

$B C D V A$ best-corrected distance visual acuity

$* P<0.05, * * * P<0.001$

age of the patients with mild chemical injuries and severe chemical injuries were $37 \pm 22$ years and $23 \pm 10$ years, respectively, which were younger than our study. Men aged 18-64 years $[4,10]$ and children aged 1-2 years [7,15] might be at the highest risk for ocular chemical injury in the workplace or residential locations due to occupational exposure and topical personal products and cleaning agents. In our study, the majority of the patients were factory workers and construction workers, which was in agreement with the findings of Bizrah and co-workers [16]: most ocular chemical injuries were work-related. Although the delay in the presentation of 133 (83.1\%) patients for medical treatment was less than $24 \mathrm{~h}$ after injury, only $64(40.0 \%)$ patients were at the consultation room in less than $3 \mathrm{~h}$. Bizrah et al. [14] also found that $87 \%$ of patients presented to the Moorfields Eye Hospital emergency department within 24 h, but they did not state the numbers of patients in more detailed time window (e.g., less than $3 \mathrm{~h}$, within 3-6 h, within 6-12 h). This suggested that the awareness of prompt treatment should be enhanced. In addition, grades II and I were the highest classification of the severity of ocular chemical injury in this study, indicating that the bulk of chemical injuries were mild to moderate.

The most common causative chemical varied by studies. The present study found that the most common causative chemical was unknown and mixed substances, followed by plural acid and alkali agents. This was in agreement with the findings of Haring et al. [4]. Haring et al. [4] found that the majority (88.2\%) of ocular chemical injuries were caused by chemical agents of unknown acidity or even from unknown chemicals, and only a few cases were classified as alkaline or acidic injuries. However, alkali agents were reported to be the most frequent causes of ocular chemical injuries in some studies $[6,17]$. This discrepancy may be due to differences in 
work content and location of injuries. Furthermore, an injury of the eye might be due to any chemical, solid, liquid, or aerosol [18]. To the best of our knowledge, this is the first study to report on the phase of the offending causative agent: the most common condition was liquid, followed by a mixture of liquid and solid, then gas, and the least was solid.

Ocular chemical injuries could result in significant damage to the ocular surface epithelium, cornea, anterior segment, and even retina and optic nerve [19]. In this study, elevated IOP was the most frequent problem $(8.4 \%)$, which was less than the findings of Tsai and co-workers [20]. An estimated incidence of secondary glaucoma was reported to be more than $20 \%$ after a severe chemical injury [20]. Lin et al. [21] found that a higher Roper-Hall grade was associated significantly with glaucoma treatment. Furthermore, long-term glaucoma medication was used in most eyes with elevated IOP in the first week after injury. The development of glaucoma might be due to synechiae and angle closure by secondary chronic inflammation, ciliary body necrosis in deeply penetrating alkali injuries, damage to the trabecular meshwork, severe uveitis, long-term steroid use, phacomorphic or phacolytic mechanisms, and contraction of the sclera [8]. Moreover, progressive optic nerve damage of glaucoma was observed in some patients with normal IOP, probably due to the subtle damage in ganglion cell layer by the alkali and the abnormal sensitivity to normal pressure [22]. Cabalag et al. [7] reported that lagophthalmos was the most common early complication, and corneal scarring was the most common late complication. Central corneal opacity or perforation was the most common complication in severe chemical injuries, and cataract and retinal attachment were also observed [6].

In the current study, the risk factors for poor visual outcomes in patients with ocular chemical burns were identified as older age, poor initial BCDVA, and irrigation $24 \mathrm{~h}$ after injury. Better initial BCDVA represented milder ocular chemical injuries, which was in agreement with the previous report that visual prognosis was better in eyes with mild chemical injuries [23]. This study confirmed the fact that immediate and extensive irrigation should be commenced immediately because it could improve prognosis. Irrigation after a chemical injury was to restore the physiological $\mathrm{pH}$ of the eye as rapidly as possible. The normal $\mathrm{pH}$ of the ocular surface after irrigation could return the aqueous $\mathrm{pH}$ in the eye to normal within $30 \mathrm{~min}$ [24]. The association between visual outcome and AMT was statistically marginal in the present study. AMT, as a temporary patch or a permanent graft, was effective in treating chemical injuries $[25,26]$. Furthermore, topical application of amniotic membrane extract was also effective in reducing inflammation and promoting reepithelization in the treatment of chemical injuries, especially for mild-to-moderate acute cases [27].

This study had several limitations. First, the retrospective nature of this study might result in incomplete patient selection and collection of data. Second, selection bias was possible because selecting the patient population according to ICD-10-CM might exclude some eligible patients. Third, the 1-month follow-up period might be short, and the visual outcome and the situation of ocular surface in these patients might still fluctuate. In addition, socioeconomic parameters, such as level of education, were not evaluated.

\section{Conclusions}

In summary, the hospitalized rate, management, and outcomes of patients with ocular chemical injuries at Jinshan District in Shanghai City, China, from January 1, 2012, to December 31, 2017, were evaluated. Patients were mainly young men engaged in factory or construction and affected by liquid chemical substances at the workplace. The risk factors for poor final BCDVA were identified as older age, poor initial BCDVA, and irrigation $24 \mathrm{~h}$ after injury, but not with the patient's gender, delay in presentation, nature of injury, condition of injury, grade, AMT, or complications. A comprehensive education program should be implemented and the use of protective eyewear should be promoted to prevent occupation-related ocular chemical injuries.

Acknowledgements This study was supported in part by the Project of Shanghai Science and Technology (Grant Nos. 17411950200; 17411950203; and 17ZR1404200), the Project of Shanghai Health and Family Planning Committee (Grant Nos. 20174Y0177 and 201640046) and the Project of Jinshan District Health and Family Planning Committee (Grant No. JSKJKTMS-2017-05). 


\section{Compliance with ethical standards}

Conflict of interest The authors declare that they have no competing interests.

Ethics approval and consent to participate This study was approved by the Ethics Committee of Jinshan Hospital of Fudan University. All procedures conformed to the tenets of the Declaration of Helsinki. Written informed consent was obtained.

Open Access This article is licensed under a Creative Commons Attribution 4.0 International License, which permits use, sharing, adaptation, distribution and reproduction in any medium or format, as long as you give appropriate credit to the original author(s) and the source, provide a link to the Creative Commons licence, and indicate if changes were made. The images or other third party material in this article are included in the article's Creative Commons licence, unless indicated otherwise in a credit line to the material. If material is not included in the article's Creative Commons licence and your intended use is not permitted by statutory regulation or exceeds the permitted use, you will need to obtain permission directly from the copyright holder. To view a copy of this licence, visit http://creativecommons.org/licenses/by/4.0/.

\section{References}

1. Merle H, Gerard M, Schrage N (2008) Ocular burns. J Fr Ophtalmol 31:723-734

2. Sharma N, Kaur M, Agarwal T et al (2018) Treatment of acute ocular chemical burns. Surv Ophthalmol 63:214-235

3. Singh P, Tyagi M, Kumar Y et al (2013) Ocular chemical injuries and their management. Oman J Ophthalmol 6:83-86

4. Haring RS, Sheffield ID, Channa R et al (2016) Epidemiologic trends of chemical ocular burns in the United States. JAMA Ophthalmol 134:1119-1124

5. Maghsoudi H, Gabraely N (2008) Epidemiology and outcome of 121 cases of chemical burn in East Azarbaijan province, Iran. Injury 39:1042-1046

6. Hong J, Qiu T, Wei A et al (2010) Clinical characteristics and visual outcome of severe ocular chemical injuries in Shanghai. Ophthalmology 117:2268-2272

7. Cabalag MS, Wasiak J, Syed Q et al (2015) Early and late complications of ocular burn injuries. J Plast Reconstr Aesthet Surg 68:356-361

8. Baradaran-Rafii A, Eslani M, Haq Z et al (2017) Current and upcoming therapies for ocular surface chemical injuries. Ocul Surf 15:48-64

9. Ghosh S, Salvador-Culla B, Kotagiri A et al (2019) Acute chemical eye injury and limbal stem cell deficiency-A prospective study in the United Kingdom. Cornea 38:8-12

10. Sher LM, Taylor DM, Robinson J et al (2012) The epidemiology of chemical eye exposures reported to the Victorian Poisons Information Centre. Eur J Emerg Med 19:389-394

11. Wang W, Zhou Y, Zeng J et al (2017) Epidemiology and clinical characteristics of patients hospitalized for ocular trauma in South-Central China. Acta Ophthalmol 95:e503e510

12. Sahraravand A, Haavisto AK, Holopainen JM et al (2017) Ocular traumas in working age adults in Finland-Helsinki ocular trauma study. Acta Ophthalmol 95:288-294

13. Wong MY, Man RE, Gupta P et al (2018) Prevalence, subtypes, severity and determinants of ocular trauma: the Singapore Chinese eye study. $\mathrm{Br} \mathrm{J}$ Ophthalmol 102:204-209

14. Bizrah M, Yusuf A, Ahmad S (2019) Adherence to treatment and follow-up in patients with severe chemical eye burns. Ophthalmol Ther 8:251-259

15. Bunker DJ, George RJ, Kleinschmidt A et al (2014) Alkalirelated ocular burns: a case series and review. J Burn Care Res 35:261-268

16. Bizrah M, Yusuf A, Ahmad S (2019) An update on chemical eye burns. Eye (Lond) 33:1362-1377

17. Macdonald EC, Cauchi PA, Azuara-Blanco A et al (2009) Surveillance of severe chemical corneal injuries in the UK. Br J Ophthalmol 93:1177-1180

18. Spector J, Fernandez WG (2008) Chemical, thermal, and biological ocular exposures. Emerg Med Clin North Am 26:125-136

19. Paschalis EI, Zhou C, Lei F et al (2017) Mechanisms of retinal damage after ocular alkali burns. Am J Pathol 187:1327-1342

20. Tsai JH, Derby E, Holland EJ et al (2006) Incidence and prevalence of glaucoma in severe ocular surface disease. Cornea 25:530-532

21. Lin MP, Eksioglu U, Mudumbai RC et al (2012) Glaucoma in patients with ocular chemical burns. Am J Ophthalmol 154:481-485

22. Cade F, Grosskreutz CL, Tauber A et al (2011) Glaucoma in eyes with severe chemical burn, before and after keratoprosthesis. Cornea 30:1322-1327

23. Brodovsky SC, McCarty CA, Snibson G et al (2000) Management of alkali burns: an 11-year retrospective review. Ophthalmology 107:1829-1835

24. Wagoner MD (1997) Chemical injuries of the eye: current concepts in pathophysiology and therapy. Surv Ophthalmol 41:275-313

25. Barreiro TP, Santos MS, Vieira AC et al (2014) Comparative study of conjunctival limbal transplantation not associated with the use of amniotic membrane transplantation for treatment of total limbal deficiency secondary to chemical injury. Cornea 33:716-720

26. Sharma N, Singh D, Maharana PK et al (2016) Comparison of amniotic membrane transplantation and umbilical cord serum in acute ocular chemical burns: a randomized controlled trial. Am J Ophthalmol 168:157-163

27. Liang L, Li W, Ling S et al (2009) Amniotic membrane extraction solution for ocular chemical burns. Clin Exp Ophthalmol 37:855-863

Publisher's Note Springer Nature remains neutral with regard to jurisdictional claims in published maps and institutional affiliations. 